\title{
Limit on the Neutrino Mass from the WMAP Three Year Data
}

\author{
Masataka Fukugita, Kazuhide Ichikawa, Masahiro Kawasaki \\ Institute for Cosmic Ray Research, \\ University of Tokyo, Kashiwa 277 8582, Japan \\ Ofer Lahav \\ Department of Physics and Astronomy, \\ University College London, Gower Street, \\ London WC1E 6BT, United Kingdom
}

(Dated: June 28, 2018)

\begin{abstract}
We derive an upper limit on the neutrino mass from the WMAP three-year data alone by employing a deterministic minimisation method based on a grid search in multi-parameter space. Assuming the flat $\Lambda \mathrm{CDM}$ model with power-law adiabatic perturbations, we find $\sum m_{\nu}<2.0 \mathrm{eV}$ in agreement with the result of the WMAP team. This result, the limit being nearly the same as that from the WMAP first-year data, means that the fundamental limit on the neutrino mass obtainable from the cosmic microwave background alone is already nearly met, as anticipated from the previous analysis. We also clarify the role of the polarisation data in deriving the limit on the neutrino mass.
\end{abstract}


In 1] it was shown that a valid limit on the neutrino mass can be derived from the cosmic microwave background (CMB) experiment alone. This contrasted to the then believed view that such a limit is obtained only when the CMB data are combined with those from largescale clustering of galaxies [2, 3] : the limit given by Tegmark et al 2] derived from the first year data of the Wilkinson Microwave Anisotropy Probe (WMAP-1) [3] is $\sum m_{\nu}<11 \mathrm{eV}$ at a $95 \%$ confidence. This implies that massive neutrinos alone may constitute the entire dark matter of the Universe. This conclusion was supported by the analysis of Elgarøy and Lahav [4]. Our limit from WMAP-1 alone, $\sum m_{\nu}<2.0 \mathrm{eV}$ (95\% CL) obtained from a deterministic grid search, contradicts with that of [2]. We ascribed this discrepancy to the possibility that Markov chain Monte Carlo used in 2] was not long enough to sample the likelihood function in the presence of strong parameter degeneracy.

The argument given by [4] is based on the well-known fact that massive neutrinos diminishes the small-scale power by free-streaming and this leads to a limit on the neutrino mass. In this argument the fact was not taken into account that massive neutrinos affect the CMB perturbations not only by the modification of the power spectrum but also in some not so obvious ways: characteristically, the acoustic length scale and the relative heights of peaks are modified. The shift of the position of the acoustic peaks and the height of the first peak alone can be absorbed into the change of cosmological parameters, but when the heights of the second and third peaks relative to the first are modified, the shifts cannot be absorbed into that of the cosmological parameters, leading to a limit on the neutrino mass. This means that a limit on neutrino mass could be obtained from the observed CMB multipoles only if neutrinos are nonrelativistic at the recombination epoch, i.e., $m_{\nu}>0.6 \mathrm{eV}$. Several numerical analyses made recently seem to support this conclusion [5, 6, 6].

At the data release of the WMAP team for their three year observations (WMAP-3) 8], they gave a limit on the neutrino mass $\sum m_{\nu}<2.0 \mathrm{eV}$ (at a $95 \%$ confidence) from their CMB data alone, using a Markov chain Monte Carlo analysis. This agrees with our conclusion in [1], where it was shown that the limit from WMAP-1, $\sum m_{\nu}<2.0 \mathrm{eV}$, could hardly be improved even with the increasing quality of the CMB data. In this report we attempt to verify this explicitly by repeating the previous analysis with the WMAP-3 data using the deterministic grid search algorithm. Incidentally, we try to elucidate the role of the polarisation data if they could tighten the constraint on the neutrino mass.

We take the flat $\Lambda$ CDM model with adiabatic perturbations of the power law spectrum. 


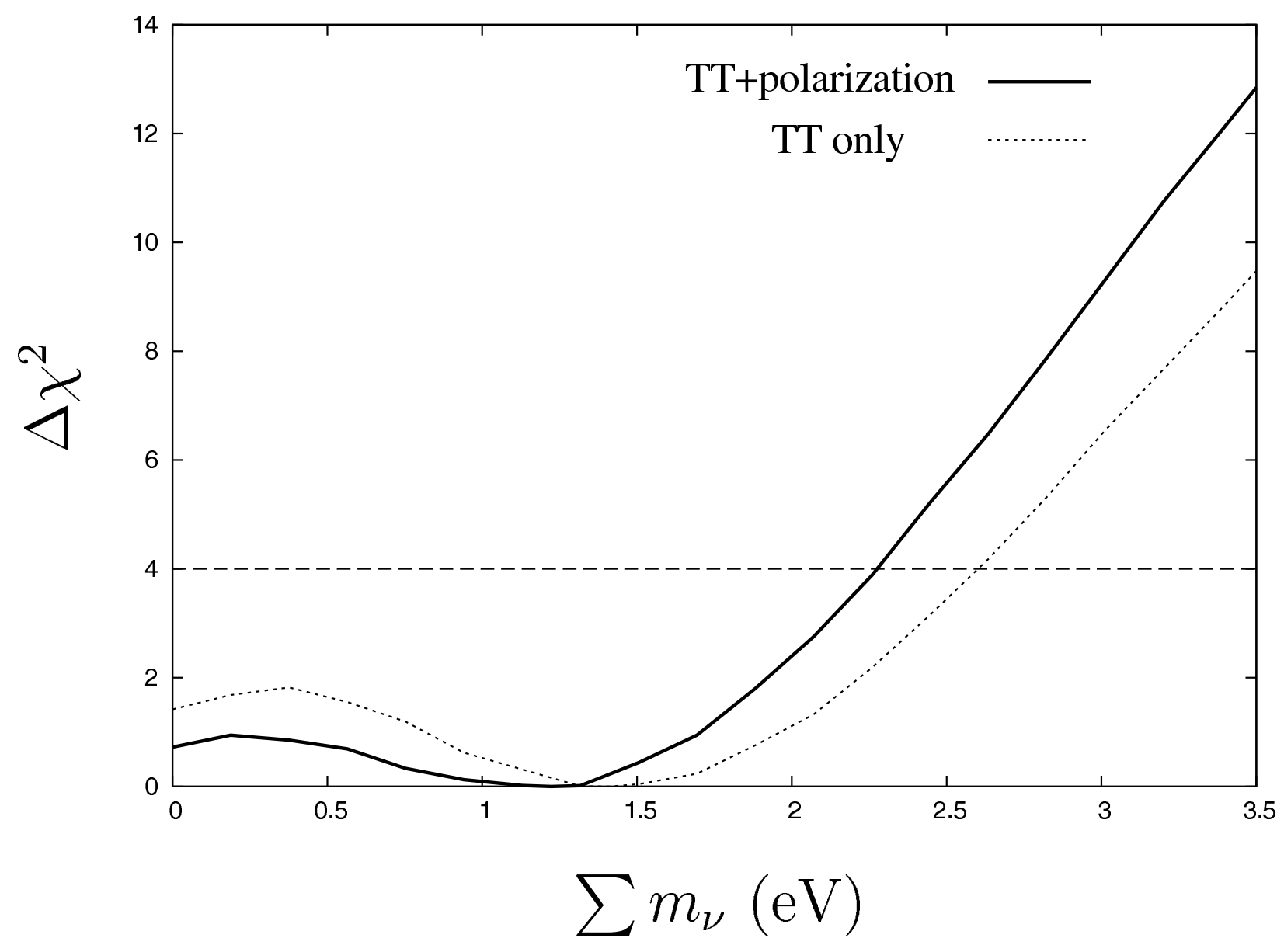

FIG. 1: The curve of $\chi^{2}\left(\omega_{\nu}\right)$ as a function of the neutrino mass summed over generations. The solid curve uses all available data from WMAP including polarisations. The dotted curve is a constraint from only temperature-temperature correlations.

The model contains 6 cosmological parameters, the mass density parameters $\left(\omega_{i}=\Omega_{i} h^{2}\right)$ for cold dark matter, baryonic matter and massive neutrinos, the normalised Hubble constant $h$, the reionization optical depth $\tau$, the scalar spectral index of primordial mass perturbations $n_{s}$ and their amplitude $A$. The neutrino mass density parameter $\omega_{\nu}$ is related to neutrino masses by $\omega_{\nu}=\sum m_{\nu} /(94.1 \mathrm{eV})$ (the Fermi distribution is assumed) and we assume three generations of massive neutrinos with a degenerate mass. Theoretical CMB power spectrum is calculated using the CMBFAST code [9] and $\chi^{2}$ by the likelihood code of the WMAP threeyear data release $10,11,12]$. We calculate the $\chi^{2}$ function for a given $\omega_{\nu}$ by minimizing it over the 6 parameters. The minimization is carried out with the grid search using the Brent method 13] extended to multi-parameters, as described in [1]. 
The result is shown in Fig. 1. The logarithm of the likelihood $-2 \ln \mathcal{L}$ for the power spectra including all TT, TE, EE and BB with the pixel-based method for low multipoles is shown by the solid line. The $\chi_{\text {eff }}^{2}$ for 3162 degrees of freedom at the vanishing neutrino mass is 1.037 for the parameters $\omega_{b}=0.0221, \omega_{m}=0.127, h=0.725, \tau=0.091$ and $n_{s}=0.957$ in agreement with the WMAP-3 analysis.

The limit corresponding to $\Delta \chi^{2}=4$ (measured from the minimum) is $\omega_{\nu} \leq 0.024$ or $\sum m_{\nu} \leq 2.3 \mathrm{eV}$. We obtain the upper limits at a $95 \%$ confidence by an integration of the likelihood function $\mathcal{L}=\exp \left\{-\Delta \chi^{2} / 2\right\}$,

$$
\omega_{\nu} \leq 0.0215 \quad \text { or } \quad \sum m_{\nu} \leq 2.0 \mathrm{eV}
$$

which corresponds to $m_{\nu}<0.67 \mathrm{eV}$. A slight difference between the limit from $\Delta \chi^{2}=4$ and likelihood integral is due to the $\chi^{2}$ curve flatter than quadratic at small neutrino masses. The actual minimum of $\chi^{2}$ occurs at a non-zero neutrino mass $\sum m_{\nu} \approx 1.3 \mathrm{eV}$, but $\chi^{2}$ relative to the vanishing neutrino mass is less than one, meaning that the preference of a finite neutrino mass is insignificant.

The limit we obtained agrees with that from the WMAP team, endorsing the validity of their Markov chain Monte Carlo analysis. We may also compare eq. (11) with that from WMAP-1 [1]: $\omega_{\nu} \leq 0.023$. The limit changes little in spite of the significantly increased accuracy of the CMB data, which agrees with the analysis given in [1]. This is close to the fundamental limit that could be obtained from the CMB alone even if the data quality would be higher. A further improvement of the limit needs external constraints, such as those from galaxy clustering.

We now briefly discuss the role of the polarisation data in deriving the limit on the neutrino mass. We show in Figure 1 above the $\chi^{2}$ curve for the TT correlation only (the dotted curve), which gives $\omega_{\nu} \leq 0.0250$ or $\sum m_{\nu} \leq 2.4 \mathrm{eV}$, slightly weaker than eq. (11). We note, however, that there is no particular sensitivity in the TE or EE correlation to the inclusion of the neutrino mass beyond that of the TT correlation data. The left column of the six panel figure, Figure 2, shows the TT, TE and EE correlators for $\omega_{\nu}=0$ and 0.02 with the other cosmological parameters fixed. In the right-column panels we rescaled the abscissa in the way that accounts for the modification of the acoustic length scale by a finite neutrino mass. The two curves almost coincide for all correlators, TT, TE and EE. The WMAP data for TT are accurate enough to marginally distinguish the two curves at 


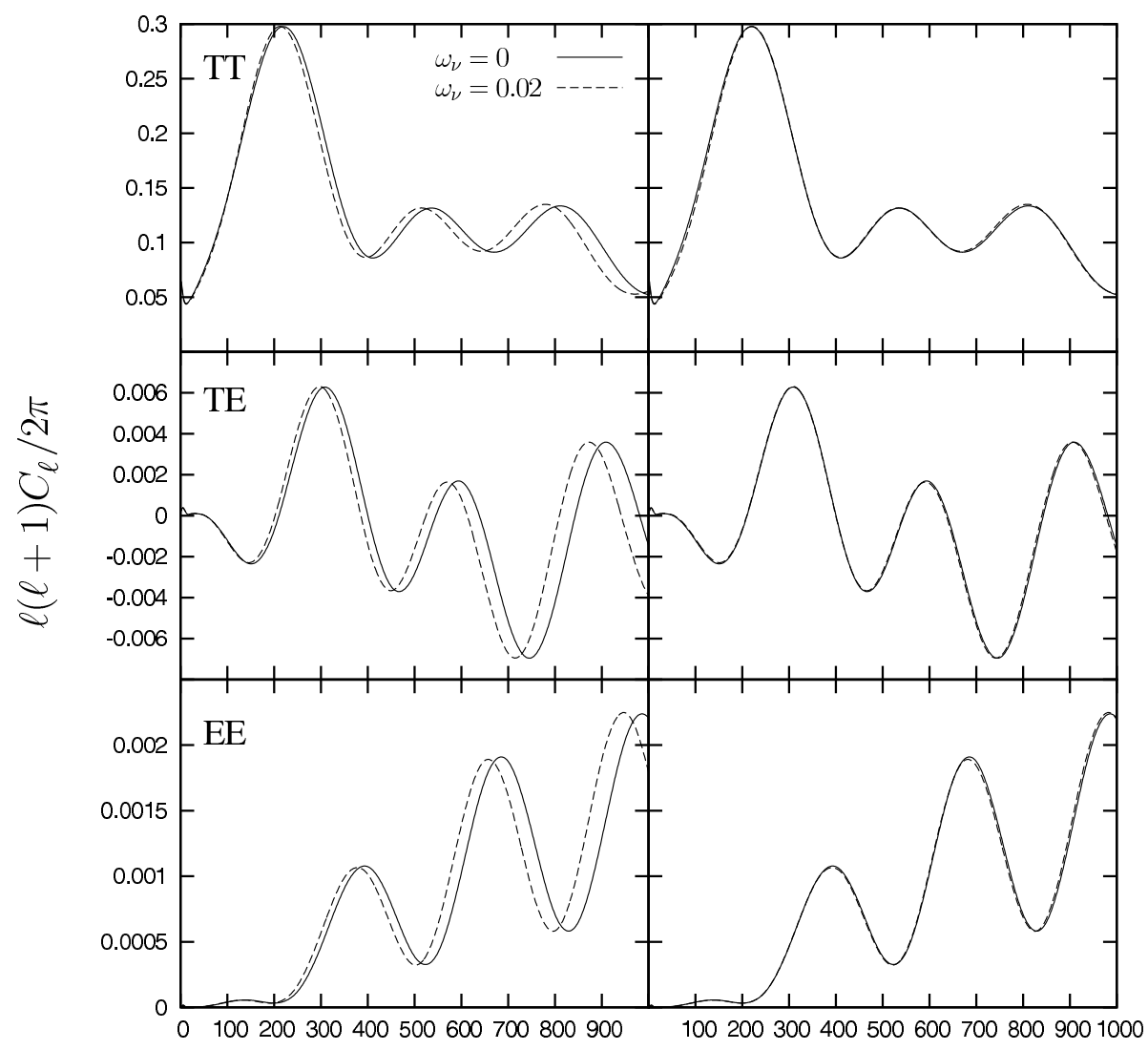

FIG. 2: TT, TE and EE correlators for three cases: $\omega_{\nu}=0$ (the solid line) and 0.02 (the dashed line) with the other parameters fixed to the WMAP mean values. In the right panels the abscissa is rescaled according to the modification of the acoustic length that varies with the neutrino mass.

approximately a 95\% confidence level. The data involving polarisation, however, do not reach this accuracy, so that they do not give new information as to the neutrino mass. We do not expect any effect of the neutrino mass that modifies polarisation other than those through the scales in acoustic dynamics. The slight improvement on the limit of the neutrino mass upon the inclusion of the polarisation data, as we saw above, arises from the tightened constraint on $\tau$. There is a significant negative correlation between $\omega_{\nu}$ and $\tau$ (see Figure $2 \mathrm{~d}$ in [1]). The absence of the polarisation data makes $\tau$ more uncertain and rather drives the $\chi^{2}$ minimum to $\tau \approx 0$, which in turn pushes the neutrino mass towards a larger value. In this way, the polarisation data serve to slightly improve the limit on the neutrino mass through a constraint on the reionisation optical depth.

Our final remark is that the limit from the CMB data alone is the most robust result in view of the uncertainties in the current large-scale galaxy clustering data. They drive the 
best fit parameter of the matter density either way depending upon whether one takes the SDSS or 2dFGRS data 8]: the limit on the neutrino mass is also sensitive to the choice of the galaxy clustering data between the two [14] as well as how much weight is given to these data. To go beyond the present neutrino mass limit it would be imperative to combine external data, but this needs proper understanding of systematic errors involved in them.

[1] K. Ichikawa, M. Fukugita and M. Kawasaki, Phys. Rev. D 71, 043001 (2005)

[2] M. Tegmark et al. [SDSS Collaboration], Phys. Rev. D 69, 103501 (2004)

[3] D. N. Spergel et al., Astrophys. J. Suppl. 148, 175 (2003)

[4] Ø. Elgarøy and O. Lahav, JCAP 0304, 004 (2003)

[5] C. J. MacTavish et al., arXiv:astro-ph/0507503.

[6] S. Hannestad, arXiv:hep-ph/0602058.

[7] J. Lesgourgues and S. Pastor, arXiv:astro-ph/0603494.

[8] D. N. Spergel et al., arXiv:astro-ph/0603449.

[9] U. Seljak and M. Zaldarriaga, Astrophys. J. 469, 437 (1996)

[10] N. Jarosik et al., arXiv:astro-ph/0603452.

[11] G. Hinshaw et al., arXiv:astro-ph/0603451.

[12] L. Page et al., arXiv:astro-ph/0603450.

[13] R. P. Brent, Algorithms for Minimization without Derivatives (Prentice-Hall, Englewood Clifs, NJ, U.S.A. 1973); see also W. H. Press, B. P. Flannery, S. A. Teukolsky and W. T. Vetterling, Numerical Recipes (Cambridge University Press, New York, 1986)

[14] M. Fukugita, Nucl.Phys.Proc.Suppl. 155, 10-17 (2006). 